\title{
Near-Optimal Network Design with Selfish Agents
}

\author{
Elliot Anshelevich ${ }^{*}$ \\ Dept. of Computer Science \\ Cornell University, Ithaca NY 14853 \\ eanshel@cs.cornell.edu \\ Éva Tardos ${ }^{\dagger}$ \\ Dept. of Computer Science \\ Cornell University, Ithaca NY 14853 \\ eva@cs.cornell.edu
}

\author{
Anirban Dasgupta \\ Dept. of Computer Science \\ Cornell University, Ithaca NY 14853 \\ adg@cs.cornell.edu \\ Tom Wexler \\ Dept. of Computer Science \\ Cornell University, Ithaca NY 14853 \\ wexler@cs.cornell.edu
}

\begin{abstract}
We introduce a simple network design game that models how independent selfish agents can build or maintain a large network. In our game every agent has a specific connectivity requirement, i.e. each agent has a set of terminals and wants to build a network in which his terminals are connected. Possible edges in the network have costs and each agent's goal is to pay as little as possible. Determining whether or not a Nash equilibrium exists in this game is NP-complete. However, when the goal of each player is to connect a terminal to a common source, we prove that there is a Nash equilibrium as cheap as the optimal network, and give a polynomial time algorithm to find a $(1+\varepsilon)$-approximate Nash equilibrium that does not cost much more. For the general connection game we prove that there is a 3 -approximate Nash equilibrium that is as cheap as the optimal network, and give an algorithm to find a $(4.65+\varepsilon)$-approximate Nash equilibrium that does not cost much more.
\end{abstract}

\section{Categories and Subject Descriptors}

F.2 [Theory of Computation]: Analysis of Algorithms and Problem complexity

\section{General Terms}

Algorithms, Economics

\section{Keywords}

network design, price of anarchy

\footnotetext{
* Research supported in part by an NSF graduate fellowship. ${ }^{\dagger}$ Research supported in part by ONR grant N00014-98-10589 .

${ }^{\ddagger}$ Research supported in part by an NSF graduate fellowship.
}

Permission to make digital or hard copies of all or part of this work for personal or classroom use is granted without fee provided that copies are not made or distributed for profit or commercial advantage and that copies bear this notice and the full citation on the first page. To copy otherwise, to republish, to post on servers or to redistribute to lists, requires prior specific permission and/or a fee.

STOC'03, June 9-11, 2003, San Diego, California, USA

Copyright 2003 ACM 1-58113-674-9/03/0006 ...\$5.00.

\section{INTRODUCTION}

Many networks, including the Internet, are developed, built, and maintained by a large number of agents (Autonomous Systems), all of whom act selfishly and have relatively limited goals. This naturally suggests a game-theoretic approach for studying both the behavior of these independent agents and the structure of the networks they generate. The stable outcomes of the interactions of non-cooperative selfish agents correspond to Nash equilibria. Considering the Nash equilibria of games modeling classical networking problems gives rise to a number of new issues. In particular, Nash equilibria in network games can be much more expensive than the best centralized design. Papadimitriou [17] uses the term price of anarchy to refer to this increase in cost caused by selfish behavior. The price of anarchy has been studied in a number of games dealing with various networking issues, such as load balancing $[6,7,15,20]$, routing $[19,21,22]$, facility location [24], and flow control [2, 8, 23]. In some cases $[19,21]$ the Nash equilibrium is unique, while in others [15] the best Nash equilibrium coincides with the optimum solution and the authors study the quality of the worst equilibrium. However, in some games the quality of even the best possible equilibria can be far from optimal (e.g. in the prisoner's dilemma). The best Nash equilibrium can be viewed as the best solution that selfish agents can agree upon, i.e. once the solution is agreed upon, the agents do not find it in their interest to deviate. Papadimitriou [17] defines the price of anarchy to study the question of how bad an equilibrium can be. We study the complementary question of how good an equilibrium can be in the context of a network design game. Schultz and Stier [22] study the ratio of the best equilibrium to the optimum, in the context of a capacitated routing game. We call this ratio the optimistic price of anarchy.

In this paper we consider a simple network design game where every agent has a specific connectivity requirement, i.e. each agent has a set of terminals and wants to build a network in which his terminals are connected. Possible edges in the network have costs and each agent's goal is to pay as little as possible. This game can be viewed as a simple model of network creation. Alternatively, by studying the best Nash equilibria, our game provides a framework for understanding those networks that a central authority could persuade selfish agents to purchase and maintain. An inter- 
esting feature of our game is that selfish agents will find it in their individual interests to share the costs of edges, and so effectively cooperate.

More precisely, we study the following network game for $N$ players, which we call the connection game. For each game instance, we are given an undirected graph $G$ with non-negative edge costs. Players form a network by purchasing some subgraph of $G$. Each player has a set of specified terminal nodes that he would like to see connected in the purchased network. With this as their goal, players offer payments indicating how much they will contribute towards the purchase of each edge in $G$. If the players' payments for a particular edge $e$ sum to at least the cost of $e$, then the edge is considered bought, which means that $e$ is added to our network and can now be used by any player. Each player would like to minimize his total payments, but insists on connecting all of his terminals. We allow the cost of any edge to be shared by multiple players. Furthermore, once an edge is purchased, any player can use it to satisfy his connectivity requirement, even if that player contributed nothing to the cost of this edge. Finding the centralized optimum of the connection game, i.e. the network of bought edges which minimizes the sum of the players' contributions, is the classic network design problem of the generalized Steiner tree $[1,12]$. We are most interested in deterministic Nash equilibria of the connection game, and in the optimistic price of anarchy, as the pessimistic price of anarchy in our game can be quite bad. In a game theoretic context it might seem natural to also consider mixed Nash equilibria when agents can randomly choose between different strategies. However, since we are modeling the construction of large-scale networks, randomizing over strategies is not a realistic option for players.

\section{Our Results}

We study deterministic Nash equilibria of the connection game, and prove bounds on the (optimistic) price of anarchy. We also explore the notion of an approximate equilibrium, and study how close to a true equilibrium a solution can be, while still purchasing the optimal network. In other words, we ask how unhappy would the agents necessarily be if they were forced to pay for the socially optimal design. We can view this as a two parameter optimization problem: we would like to have a solution with cost close to the minimum possible cost, and where users would not have large incentives to deviate. Finally, we examine how difficult it is to find equilibria at all.

- In Section 3 we consider the special case when the goal of each player is to connect a single terminal to a common source. We prove that in this case, there is a Nash equilibrium, the cost of which is equal to the cost of the optimal network. In other words, with a single source and one terminal per player, the optimistic price of anarchy is 1 . Furthermore, given an $\varepsilon>0$ and an $\alpha$-approximate solution to the optimal network, we show how to construct in polynomial time an $(1+\varepsilon)$-approximate Nash equilibrium (players only benefit by a factor of $(1+\varepsilon)$ in deviating) whose total cost is within a factor of $\alpha$ to the optimal network.

We generalize these results in two ways. First, we can extend the results to the case when the graph is directed and players seek to establish a directed path from their terminal to the common source. Note that problems in directed graphs are often significantly more complicated than their undirected counterparts $[5,10]$. Second, players do not have to insist on connecting their terminals at all cost, but rather each player $i$ may have a maximum $\operatorname{cost} \max (i)$ that he is willing to pay, and would rather stay unconnected if his cost exceeds $\max (i)$.

- In Section 4 we consider the general case, when players may want to connect more than 2 terminals, and they do not necessarily share a single source node. In this case, there may not exist a deterministic Nash equilibrium. When deterministic Nash equilibria do exist, the costs of different equilibria may differ by as much as a factor of $N$, the number of players, and even the optimistic price of anarchy may be nearly $N$. However, in Section 4 we prove that there is always a 3 -approximate equilibrium that pays for the optimal network. Furthermore, we show how to construct in polynomial time a $(4.65+\varepsilon)$-approximate Nash equilibrium whose total cost is within a factor of 2 to the optimal network.

- Finally, in Section 5 we show that determining whether or not a Nash equilibrium exists is NP-complete when the number of players is part of the input. We also show that the same problem is poly-time solvable for 2 players that have two terminals each. Since there are only a polynomial number of Nash equilibrium structures, the algorithm simply enumerates these.

\section{Related Work}

We view our game as a simple model of how different service providers build and maintain the Internet topology. We use a game theoretic version of network design problems considered in approximation algorithms [12]. Fabrikant et al [9] study a different network creation game. Network games similar to that of [9] have also been studied for modeling the creation and maintenance of social networks $[4,13]$. In the network game considered in $[4,9,13]$ each agent corresponds to a single node of the network, and agents can only buy edges adjacent to their nodes. This model of network creation seems extremely well suited for modeling the creation of social networks. However, in the context of communication networks like the Internet, agents are not directly associated with individual nodes, and can build or be responsible for more complex networks. There are many situations where agents will find it in their interest to share the costs of certain expensive edges. An interesting feature of our model that does not appear in $[4,9,13]$ is that we allow agents to share costs in this manner. To keep our model simple, we assume that each agent's goal is to keep his terminals connected, and agents are not sensitive to the length of their connecting path.

Jain and Vazirani [14] study a cost-sharing game closely related to the single source network creation game considered in Section 3. They assume that each player $i$ has a utility $u_{i}$ for belonging to the Steiner tree (the maximum they would be willing to pay). Cost-sharing games assume that there is a central authority that designs and maintains the network, and decides appropriate cost-shares for each agent. The only role of the agents is to report their utility. The goal of the cost-sharing method is to pay for the network built, and produce cost-shares such that selfish agents are truthful, i.e. do not find it in their interest to misreport their utility (in hopes of being included in the network at a smaller cost). Jain and Vazirani give a truthful mechanism 
to share the cost of the minimum spanning tree, which is a 2-approximation for the Steiner tree problem.

In contrast, in our game the agents contribute to individual edges directly, rather than contributing money to a central authority. Therefore, each player is free to choose his own paths, instead of having the central authority specify paths for each player. A game with low price of anarchy is one where any stable outcome of selfish interactions of agents is of low cost, without any help from a central authority.

In terms of central coordination, the optimistic price of anarchy can be viewed as lying half way between the traditional price of anarchy and cost-sharing. Selecting the best Nash equilibrium effectively assumes some coordination: a central authority that guides the players to the best Nash equilibrium. However, once the players settle on such an equilibrium strategy, it is self enforcing: no player finds it in his interest to deviate. The role of the central authority is limited to guiding the players to a better Nash equilibrium.

\section{MODEL AND BASIC RESULTS}

The Connection Game. We now formally define the connection game for $N$ players. Let an undirected graph $G=$ $(V, E)$ be given, with each edge $e$ having a nonnegative cost $c(e)$. Each player $i$ has a set of terminal nodes that he must connect. The terminals of different players do not have to be distinct. A strategy of a player is a payment function $p_{i}$, where $p_{i}(e)$ is how much player $i$ is offering to contribute to the cost of edge $e$. Any edge $e$ such that $\sum_{i} p_{i}(e) \geq c(e)$ is considered bought, and $G_{p}$ denotes the graph of bought edges with the players offering payments $p=\left(p_{1}, \ldots, p_{N}\right)$. Since each player must connect his terminals, all of the player's terminals must be connected in $G_{p}$. However, each player tries to minimize his total payments, $\sum_{e \in E} p_{i}(e)$.

A Nash equilibrium of the connection game is a payment function $p$ such that, if players offer payments $p$, no player has an incentive to deviate from his payments. This is equivalent to saying that if $p_{j}$ for all $j \neq i$ are fixed, then $p_{i}$ minimizes the payments of player $i$. A $(1+\varepsilon)$-approximate Nash equilibrium is a function $p$ such that no player $i$ could decrease his payments by more than a factor of $1+\varepsilon$ by deviating, i.e. by using a different payment function $p_{i}{ }^{\prime}$.

Basic Results. Here we present several useful properties of Nash equilibria in the connection game. Suppose we have a Nash equilibrium $p$, and let $T^{i}$ be the smallest tree in $G_{p}$ connecting all terminals of player $i$. It easily follows from the definitions that (1) $G_{p}$ is a forest, (2) each player $i$ only contributes to costs of edges on $T^{i}$, and (3) each edge is either paid for fully or not at all.

It is not always the case that selfish agents can agree to pay for a network. There are instances of the connection game that have no deterministic Nash equilibria. In Figure 1 , there are 2 players, one wishing to connect node $s_{1}$ to node $t_{1}$, and the other $s_{2}$ to $t_{2}$. Now suppose that there exists a Nash equilibrium $p$. By Property 1 above, $G_{p}$ must be a forest, so assume without loss of generality it consists of the edges $a, b$, and $c$. By Property 2, player 1 only contributes to edges $a$ and $b$, and player 2 only contributes to edges $b$ and $c$. This means that edges $a$ and $c$ must be bought fully by players 1 and 2, respectively. At least one of the two players must contribute a positive amount to edge $b$. How- ever, neither player can do that in a Nash equilibrium, since then he would have an incentive to switch to the strategy of only buying edge $d$ and nothing else, which would connect his terminals with his payments totalling only 1.

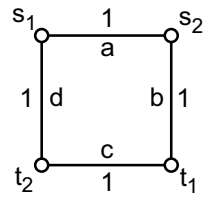

Figure 1: A game with no Nash equilibria.

We have now shown that Nash equilibria do not have to exist. However, when they exist, how bad can these Nash equilibria be? As mentioned above, the price of anarchy is the ratio of the worst (most expensive) Nash equilibrium and the optimal centralized solution. In the connection game, the price of anarchy is at most $N$, the number of players. This is simply because if the worst Nash equilibrium $p$ costs more than $N$ times OPT, the cost of the optimal solution, then there must be a player whose payments in $p$ are strictly more than OPT, so he could deviate by purchasing the entire optimal solution by himself, and connect his terminals with smaller payments than before. More importantly, there are cases when the price of anarchy actually equals $N$, so the above bound is tight. This is demonstrated with the following example. Suppose there are $N$ players, and $G$ consists of nodes $s$ and $t$ that are joined by 2 disjoint paths, one of cost 1 and one of cost $N$. Each player has a terminal at $s$ and $t$. Then, the worst Nash equilibrium has each player contributing 1 to the long path, and has a cost of $N$. The optimal solution here has a cost of only 1 , so the price of anarchy is $N$. Therefore, the price of anarchy could be very high in the connection game. However, notice that in this example the best Nash equilibrium (in which each player pays $\frac{1}{N}$ for the cheap path) has the same cost as the optimal centralized solution. We have now shown that the price of anarchy can be very large in the connection game, but the optimistic price of anarchy remains worth considering, since the above example shows that it can differ from the (traditional) price of anarchy by as much as a factor of $N$.

\section{SINGLE SOURCE GAMES}

In this section we define a class of games in which there is always a Nash equilibrium, and the optimistic price of anarchy is 1 . Furthermore, we show how we can use an approximation to the centrally optimal network to construct a $(1+\epsilon)$-approximate Nash equilibrium in poly-time, for any $\epsilon>0$.

Definition 3.1 A single source game is a game in which all players share a common terminal $s$, and each player $i$ has exactly one other terminal $t_{i}$.

We will now show that the optimistic price of anarchy is 1 in single source games. To do this, we must argue that there is a Nash equilibrium that purchases $T^{*}$, the minimum cost Steiner tree on the players' terminal nodes. There are a number of standard cost-sharing methods for sharing the cost of a tree among the terminals. The two most commonly studied methods are the Shapley value and the Marginal 
Cost mechanisms [11]. The Marginal Cost (or VCG) mechanisms are very far from being budget balanced, i.e. the agents do not pay for even a constant fraction of the tree built. The Shapley value mechanism is budget balanced: the cost of each edge is evenly shared by the terminals that use the edge for their connection (i.e., the terminals in the subtree below the edge $e$ ). However, the mechanism does not lead to a Nash equilibrium in our game: some players can have cheaper alternate paths, and hence benefit by deviating. Jain and Vazirani [14] give a truthful budget balanced cost-sharing mechanism to pay for the minimum spanning tree, which is a 2-approximate budget balanced mechanism for the Steiner tree problem. However, it is only a 2-approximation, and the cost-shares are not associated with edges that the agents use. Here we will show that while the traditional Steiner tree cost-sharing methods do not lead to a Nash equilibrium, such a solution can be obtained.

Theorem 3.2 In any single source game, there is a Nash equilibrium that purchases $T^{*}$, a minimum cost Steiner tree on all players' terminal nodes.

Proof. Given $T^{*}$, we present an algorithm to construct payment strategies $p$. We will view $T^{*}$ as being rooted at $s$. Let $T_{e}$ be the subtree of $T^{*}$ disconnected from $s$ when $e$ is removed. We will determine payments to edges by considering edges in reverse breadth first search order. We determine payments to the subtree $T_{e}$ before we consider edge $e$. In selecting the payment of agent $i$ to edge $e$ we consider $c^{\prime}$, the cost that player $i$ faces if he deviates in the final solution: edges $f$ in the subtree $T_{e}$ are considered to cost $p_{i}(f)$, edges $f$ not in $T^{*} \operatorname{cost} c(f)$, while all other edges cost 0 . We never allow $i$ to contribute so much to $e$ that his total payments exceed his cost of connecting $t_{i}$ to $s$.

\section{Algorithm 3.3}

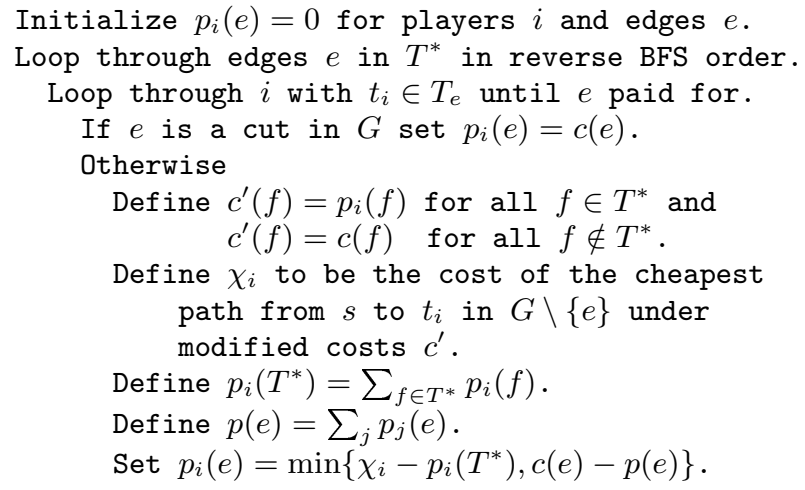

We first claim that if this algorithm terminates, the resulting payment forms a Nash equilibrium. We ensure that player $i$ 's contributions to edges are always less than his cost of connecting $t_{i}$ to $s$. Therefore it is never in player $i$ 's interest to deviate. Since this is true for all players, $p$ is a Nash equilibrium.

We will now prove that this algorithm succeeds in paying for $T^{*}$. In particular, we need to show that for any edge $e$, the players with terminals in $T_{e}$ will be willing to pay for $e$. Assume the players are unwilling to buy an edge $e$. Then each player has some path that explains why it can't contribute more to $e$. We can use a carefully selected subset of these paths to modify $T^{*}$, forming a cheaper tree that spans all terminals and doesn't contain $e$. This would clearly contradict our assumption that $T^{*}$ had minimum cost.

Define player $i$ 's alternate path $A_{i}$ to be the path of cost $\chi_{i}$ found in Algorithm 3.3, as shown in Figure 2(a). If there is more than one such path, choose the path that includes as many ancestors of $t_{i}$ in $T_{e}$ as possible before including edges outside of $T^{*}$. To show that all edges in $T^{*}$ are paid for, we need the following technical lemma concerning the structure of alternate paths.

Lemma 3.4 Suppose $A_{i}$ is $i$ 's alternate path at some stage of the algorithm. Then there are two nodes $v$ and $w$ on $A_{i}$, such that all edges on $A_{i}$ from $t_{i}$ to $v$ are in $T_{e}$, all edges between $v$ and $w$ are in $E \backslash T^{*}$, and all edges between $w$ and $s$ are in $T^{*} \backslash T_{e}$.

Proof. Once $A_{i}$ reaches a node $w$ in $T^{*} \backslash T_{e}$, all subsequent nodes of $A_{i}$ will be in $T^{*} \backslash T_{e}$, as all edges $f$ in $T^{*} \backslash T_{e}$ have cost $c^{\prime}(f)=0$ and the source $s$ is in $T^{*} \backslash T_{e}$. Thus, suppose $A_{i}$ begins with a path $P_{1}$ in $T_{e}$, followed by a path $P_{2}$ containing only edges not in $T^{*}$, before reaching a node $x$ in $T_{e}$, as shown in Figure 2(b). Let $y$ be the lowest common ancestor of $x$ and $t_{i}$ in $T_{e}$. Observe that $P_{1}$ is strictly below $y$. Define $P_{3}$ to be the path from $t_{i}$ to $y$ in $T_{e}$, and define $P_{4}$ to be the path from $y$ to $x$ in $T_{e}$. We now show that under the modified cost function $c^{\prime}, P_{3} \cup P_{4}$ is at least as cheap as $P_{1} \cup P_{2}$. Since $P_{1} \cup P_{2}$ includes a higher ancestor of $t_{i}$ than $A_{i}$ (namely $y$ ), this contradicts our choice of $A_{i}$.

Consider the iterations of the algorithm during which player $i$ could have contributed to edges in $P_{3}$. At each of these steps the algorithm computes a cheapest path from $t_{i}$ to $s$. At any time, player $i$ 's payments are upper bounded by the modified cost of his alternate path, which is in turn upper bounded by the modified cost of any path, in particular $A_{i}$. Furthermore, at each of these steps the modified costs of all edges in $A_{i}$ above $x$ are 0 . Therefore $i$ 's contribution to $P_{3}$ is always at most the modified cost of $P_{1} \cup P_{2}$. The modified cost of $P_{4}$ is always 0 , as none of the edges in $P_{4}$ are on player $i$ 's path from $t_{i}$ to $s$ in $T^{*}$. Together these imply that $c^{\prime}\left(P_{3} \cup P_{4}\right)=c^{\prime}\left(P_{3}\right) \leq c^{\prime}\left(P_{1} \cup P_{2}\right)$.

Thus, players' alternate paths may initially use some edges in $T_{e}$, but subsequently will exclusively use edges outside of $T_{e}$. We use this fact in the following lemma.

\section{Lemma 3.5 Algorithm 3.3 pays for all edges in $T^{*}$.}

Proof. Suppose that for some edge $e$, after all players have contributed to $e, p(e)<c(e)$. For each player $i$, consider the longest subpath of $A_{i}$ containing $t_{i}$ and only edges in $T_{e}$. Call the highest ancestor of $t_{i}$ on this subpath $i$ 's deviation point, denoted $d_{i}$. Note that it is possible that $d_{i}=t_{i}$. Let $D$ be a minimum set of deviation points such that every terminal in $T_{e}$ has an ancestor in $D$.

Suppose every player $i$ with a terminal $t_{i}$ in $D$ deviates to $A_{i}$, as shown in Figure 2(c), paying his modified costs to each edge. Any player $i$ deviating in this manner does not increase his total expenditure, as player $i$ raised $p_{i}(e)$ until $p_{i}$ matched the modified cost of $A_{i}$. The remaining players leave their payments unchanged.

We claim that now the edges bought by players with terminals in $T_{e}$ connect all these players to $T^{*} \backslash T_{e}$. To see this, first consider any edge $f$ below a deviation point $d_{i}$ in $D$. By Lemma 3.4, player $i$ is the only deviating player who 


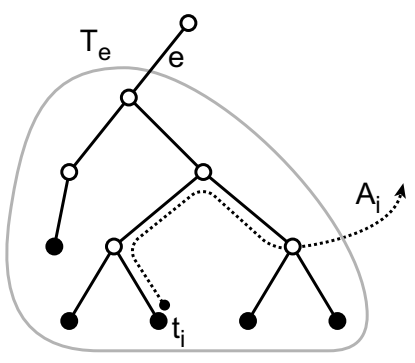

(a)

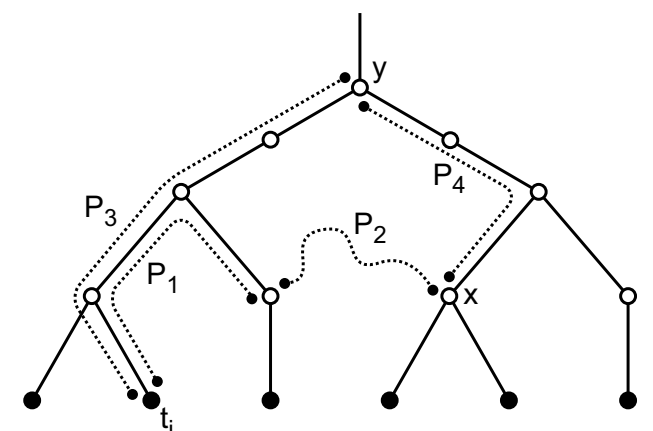

(b)

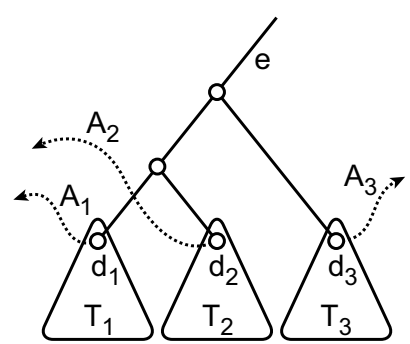

(c)

Figure 2: Alternate paths in single source games.

could have been contributing to $f$. If $i$ did contribute to $f$, then $f$ must be on the unique path from $t_{i}$ to $d_{i}$ in $T_{e}$. But by the definition of $d_{i}$, this means that $f$ is in $A_{i}$. Thus player $i$ will not change his payment to $f$.

Define $T_{i}$ to be the subtree of $T_{e}$ rooted at $d_{i}$. We have shown that all edges in $T_{i}$ have been bought. By Lemma 3.4, we know that $A_{i}$ consists of edges in $T_{i}$ followed by edges in $E \backslash T$ followed by edges in $T^{*} \backslash T_{e}$. By the definition of $c^{\prime}$, the modified cost of those edges in $E \backslash T^{*}$ is their actual cost. Thus $i$ pays fully for a path connecting $T_{i}$ to $T^{*} \backslash T_{e}$.

We have assumed that the payments generated by the algorithm for players with terminals in $T_{e}$ were not sufficient to pay for those terminals to connect to $T^{*} \backslash T_{e}$. However, without increasing any players' payments, we have bought a subset of edges that connects all terminals in $T_{e}$ to $T^{*} \backslash T_{e}$. This contradicts the optimality of $T^{*}$. Thus the algorithm runs to completion.

Since we have also shown that the algorithm always produces a Nash equilibrium, this concludes the proof of the theorem.

We have shown that the optimistic price of anarchy in a single source game is 1 . However, the algorithm for finding an optimal Nash equilibrium requires a minimum cost Steiner tree as input. Since this is computationally infeasible, we present the following result.

Theorem 3.6 Suppose we have a single source game and an $\alpha$-approximate minimum cost Steiner tree $T$. Then for any $\varepsilon>0$, there is a poly-time algorithm that returns a $(1+\varepsilon)$-approximate Nash equilibrium on a Steiner tree $T^{\prime}$, where $c\left(T^{\prime}\right) \leq c(T)$.

Proof Sketch. The proof of Theorem 3.2 suggests such an algorithm that forms a cheaper tree whenever a Nash equilibrium cannot be found. To ensure polynomial-time convergence, we force the algorithm to make only substantial improvements. See the full version of this paper for further details [3].

\section{Extensions}

Both theorems 3.2 and 3.6 can be proven for the case where our graph $G$ is directed, and players wish to purchase paths from $t_{i}$ to $s$. The one difficulty arises from the fact that in proving Lemma 3.4, we assume that paths, in particular $P_{4}$, can be traversed in either direction. In the directed case, this is no longer necessarily so. We can get around this problem with a more complicated argument, showing that if $e$ cannot be paid for, then by removing segments of alternate paths between pairs of subtrees $T_{i}$ and $T_{j}$, we can connect all terminals in $T_{e}$ at lower cost.

Once we have shown that our theorems apply in the directed case, we can extend our model and give each player $i$ a maximum cost $\max (i)$ beyond which he would rather pay nothing and not connect his terminals. It suffices to make a new terminal $t_{i}^{\prime}$ for each player $i$, with a directed edge of cost 0 to $t_{i}$ and a directed edge of cost $\max (i)$ to $s$.

\section{GENERAL CONNECTION GAMES}

In this section we deal with the general case of players that can have different numbers of terminals and do not necessarily share the same source terminal. As stated before, in this case the price of anarchy can be as large as $N$, the number of players. However, even the optimistic price of anarchy may be quite large in this general case.

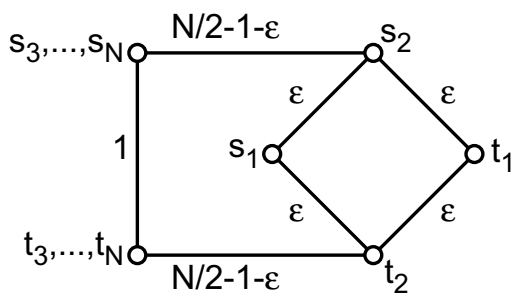

Figure 3: A game with high optimistic price of anarchy.

Consider the graph illustrated in Figure 3, where each player $i$ owns terminals $s_{i}$ and $t_{i}$. The optimal centralized solution has cost $1+3 \varepsilon$. If the path of cost 1 was bought, each player $i>2$ will not want to pay for any $\varepsilon$ edges, and therefore the situation of players 1 and 2 reduces to the example in Section 2 of a game with no Nash equilibria. Therefore, any Nash equilibrium must purchase the path of cost $N-2$. In fact, if each player $i>2$ buys $\frac{1}{N-2}$ of this path, then we have a Nash equilibrium. Therefore, for any $N>2$, there exists a game with the optimistic price of anarchy being nearly $N-2$.

Because of this, we cannot hope to provide cheap Nash equilibria for the multi-source case. Therefore, we consider 
how cheap $\alpha$-approximate Nash equilibria with small $\alpha$ can be, and obtain the following result.

Theorem 4.1 For any optimal centralized solution $T^{*}$, there exists a 3-approximate Nash equilibrium such that the purchased edges are exactly $T^{*}$.

Let $T^{*}$ be an optimal centralized solution, which we know is a forest. Define a segment of a tree $T$ as a path $P \subseteq T$ such that all interior nodes of $P$ have degree 2. For simplicity of the proof, we assume that every segment of $T^{*}$ is a single edge, since this proof is easily extendable to the general case where this need not hold. We also assume that $T^{*}$ is a tree, since otherwise we can apply this proof to each component of $T^{*}$. Let $T^{i}$ be the unique smallest subtree of $T^{*}$ that connects all terminals of player $i$.

Definition 4.2 A connection set $S$ of player $i$ is a subset of edges of $T^{i}$ such that for each connected component $C$ of the graph $T^{*} \backslash S$, we have that either

(1) any player that has terminals in $C$ has all of his terminals in $C$, or

(2) player $i$ has a terminal in $C$.

Intuitively, a connection set $S$ is a set such that if we removed it from $T^{*}$ and then somehow connected all the terminals of $i$, then all the terminals of all players are still connected in the resulting graph. Since $T^{*}$ is optimal, this means that any connection set $S$ with respect to $i$ must be cheaper than any deviation of $i$ from a strategy where $i$ pays for $S$. We now have the following lemma, the proof of which follows directly from the definition of a connection set. This lemma basically says that if each player buys at most $\alpha$ connection sets in full, then we have an $\alpha$-approximate Nash equilibrium.

Lemma 4.3 Let $p$ be a payment function purchasing $T^{*}$ that obeys the following properties.

(1) If $p(e)>0$, then $e$ is bought fully by a single player.

(2) Each player $i$ only buys edges that lie in his tree $T^{i}$.

If the set of edges that each player buys is a union of at most $\alpha$ connection sets, then $p$ is an $\alpha$-approximate Nash equilibrium.

Proof of Theorem 4.1. Now all that we need to prove Theorem 4.1 is a payment scheme for arbitrary games such that the conditions in Lemma 4.3 hold with $\alpha=3$. We now exhibit such a scheme on the edges of $T^{*}$. First, each player $i$ pays for the edges belonging only to $T^{i}$ and no other tree $T^{j}$. This is clearly a connection set, so we want each player to pay for at most 2 more. We can contract the edges now paid for, forming a new tree $T^{*}$ that the players must pay for, and on which each edge belongs to at least two different $T^{i}$ s. For convenience, we will now talk of terminals making payments instead of players. The total payment of a player is just the sum of the payments of his terminals.

Now we recursively assign terminals to the edges of $T^{*}$. Each edge is assigned a terminal that pays for it. At the end of each phase of the recursion, we generate a set of directed paths $R$ to be paid for during the following phase. Each of these paths starts at some terminal $t$, and ends at a node of a path paid for in the previous phase. We call such a path $R(t)$, since for each terminal there is at most 1 path starting at that terminal, and we call the last node of this path $r(t)$.
Figure 4(a) shows a decomposition of $T^{*}$ into these paths after this recursion is done. Initially, select $R(t)$ to be a path from an arbitrary terminal $t$ to another terminal of the same player in $T^{i}$, direct this path away from $t$, and set $R=\{R(t)\}$. Each phase proceeds as follows.

Step 1: Consider the set of directed paths $R$ generated by the previous phase. For each path $R(s)$ in this set, do the following. Let $v_{1}, v_{2}, \ldots$ be the nodes of $R(s)$, ordered in the direction away from $s$ (so that $s=v_{1}$ ). We assumed that $T^{*}$ has no non-terminal nodes of degree 2 , so each $v_{k}$ must be either a terminal or have edges not in $R(s)$ incident to it. Consider the subtree rooted at $v_{k}$ obtained by cutting edges $\left(v_{k-1}, v_{k}\right)$ and $\left(v_{k}, v_{k+1}\right)$. Define $S_{k}$ to be the set of terminals in this subtree such that the player $i$ who owns them has terminals outside this subtree as well, i.e. either $\left(v_{k-1}, v_{k}\right)$ or $\left(v_{k}, v_{k+1}\right)$ is in $T^{i}$. Do not include $s$ in $S_{1}$, and set this set to be empty for $r(s)$. We form a path $Q(t)$ for each terminal $t \in S_{k}$ that belongs to $i$ as follows.

- If $i$ does not own $s$, pick the smallest $\ell>k$ such that $S_{\ell}$ contains a terminal of player $i$.

- If such a node $v_{\ell}$ exists, set $Q(t)$ to be the path from $v_{k}$ to $v_{\ell}$.

- If no such node $v_{\ell}$ exists, and $T^{i}$ contains $r(s)$, set $Q(t)$ to be the path from $v_{k}$ to $r(s)$.

- If no such node $v_{\ell}$ exists, and $T^{i}$ does not contain $r(s)$, set $Q(t)$ to be the path from $v_{k^{\prime}}$ to $v_{k}$, where $v_{k^{\prime}}$ is the first node of $R(s)$ such that $S_{k^{\prime}}$ contains a terminal belonging to player $i$.

- If $i$ owns $s$, pick the largest $\ell<k$ such that $S_{\ell}$ contains a terminal of player $i$.

- If such a node $v_{\ell}$ exists, set $Q(t)$ to be the path from $v_{\ell}$ to $v_{k}$.

- If no such node $v_{\ell}$ exists, set $Q(t)$ to be the path from $s$ to $v_{k}$.

Figure 4(b) illustrates what the paths $Q(t)$ for terminals $t$ of $i$ look like if $i$ does not own $s$ and $T^{i}$ contains $r(s)$. In this figure, the terminals $t_{1}$ through $t_{4}$ are all the terminals of $i$ in any of the sets $S_{k}$. Figure 4(c) shows the same thing in the case that $i$ owns $s$.

Definition 4.4 A $\underline{\text { link }} L$ is a maximal set of edges of $R(s)$ such that for every edge $e \in L$, the set of paths $Q(t)$ that contain $e$ is exactly the same, for $t \in \cup_{v_{k} \in R(s)} S_{k}$.

A link $L$ is really a connection set of any terminal $t$ with $L \subseteq Q(t)$, since if we take out $L$ and add a path connecting endpoints of $Q(t)$, then all of the endpoints of all other paths $Q\left(t^{\prime}\right)$ remain connected. We would like to choose exactly one terminal $t$ from each set $S_{k}$ and have these pay for the path $R(s)$ together, with each one paying for at most 1 link $L$, and with $L \subseteq Q(t)$. We do this by constructing the following bipartite graph $(A, B)$.

Step 2: Let $A$ have a node for each link in $R(s)$, and let $B$ be the nodes of $R(s)$. Form an edge between a node $v_{k} \in B$ and node $L \in A$ if there exists some terminal $t \in S_{k}$ such that $L \subseteq Q(t)$. For $X \subseteq A$, define $\partial(X)$ to be the set of nodes in $B$ that $X$ has edges to. According to Hall's 


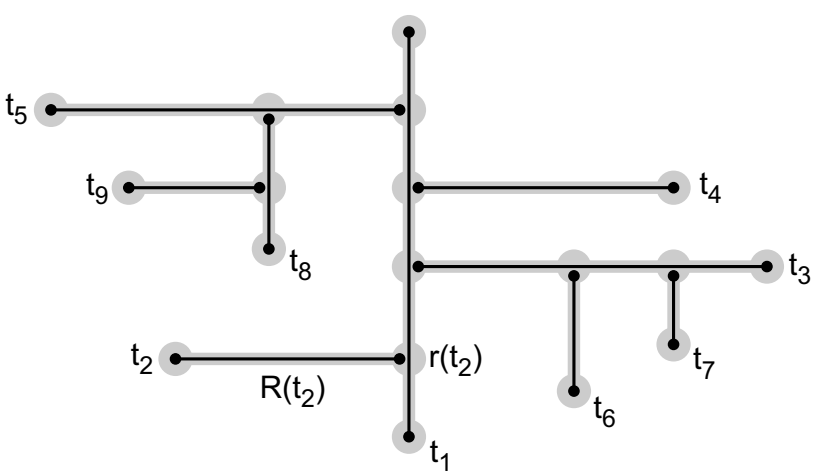

(a)

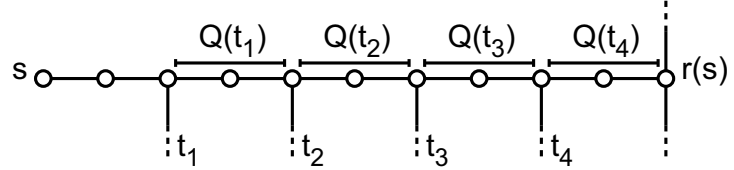

(b)

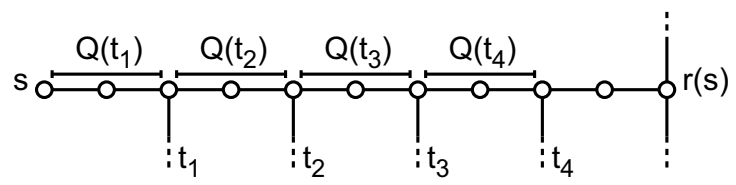

(c)

Figure 4: (a) A decomposition of $T^{*}$ into paths $R(t)$; (b)(c) The paths $Q(t)$ for a single player $i$.

Matching Theorem, there exists a matching in this bipartite graph with all nodes of $A$ incident to an edge of the matching if for each set $X \subseteq A,|\partial(X)| \geq|X|$. Arrange the edges of the links of $X$ in the order they appear in $R(s)$. We want to show that between every link of $X$, there appears a node belonging to $\partial(X)$.

Consider some edge $e$ of $X$ that is not the first one in $R(s)$. Suppose this edge belongs to link $L$, and the previous edge $e^{\prime}$ in $X$ to some link $L^{\prime}$. Since these are different links, there must be some path $Q(t)$, that either ended or began between $e^{\prime}$ and $e$, with $t \in S_{k}$ and $v_{k} \in \partial(X)$. Suppose it ended there. If $t$ belongs to the same player as $s$, then $v_{k}$ is between $e^{\prime}$ and $e$ by definition of $Q(t)$. Otherwise, there must be some terminal $t^{\prime}$ belonging to the same player as $t$ such that $Q\left(t^{\prime}\right)$ begins at the place where $Q(t)$ ends. If $Q\left(t^{\prime}\right)$ contains $e$, then we are done. Otherwise, continue this argument with $t^{\prime}$ instead of $t$, until $Q\left(t^{\prime}\right)$ contains either $e$ or $e^{\prime}$, one of which must happen by construction of $Q(t)$. Therefore, we obtain a node $v_{k}$ in $\partial(X)$ located between $e^{\prime}$ and $e$. The case of $Q(t)$ beginning between $e^{\prime}$ and $e$ is similar.

Let $L$ be the first link of $X$ that appears in $R(s)$, and suppose player $i$ owns $s$. It cannot be that $L$ belongs to a single path $Q(t)$ where $i$ owns $t$. Otherwise, this would mean that $L \in T^{i}$ but in no other tree $T^{j}$, and these edges have already been paid for and contracted. Therefore, there must be some $Q(t)$ containing $L$ such that $t$ belongs to a different player than $s$. By construction of $Q(t), t \in S_{k}$ for some $v_{k}$ that comes before $L$. This means that there is a node of $\partial(X)$ before $L$.

Therefore, $|X| \leq|\partial(X)|$, and so we can assign a terminal $t_{k} \in S_{k}$ to each node $v_{k}$ such that these terminals pay for all the links of $R(s)$ while each paying for at most 1 link, with that link in $Q\left(t_{k}\right)$. If $t_{k}$ pays for link $L$, assign the payment of $L$ to the player who owns $t_{k}$.

Step 3: Finally, we must generate the set of paths $R$ for the next phase. For each terminal $t_{k}$ chosen in Step 2, let $R\left(t_{k}\right)$ be the path from $t_{k}$ to $v_{k}$ as above. Together, these paths compose $R$.

We have now generated a payment $p$ that satisfies all of the conditions of Lemma 4.3. All that is left to prove Theorem 4.1 is that with this payment, $\alpha=3$. Since the edges that belong only to $T^{i}$ and no other tree $T^{j}$ form a connection set, we prove this inductively by showing that the set of edges paid for by each player's terminals in the above scheme is a union of at most 2 connection sets. The inductive hypothesis is the following:

Case 1: If $T^{i}$ is entirely contained in $T(s)$, then the edges paid for by terminals of $i$ are the union of at most 2 connection sets.

Case 2: If $T^{i}$ is not contained in $T(s)$, and $i$ owns $s$, then the edges paid for by terminals of $i$ in $T(s)$ is a single connection set, and $r(s)$ is connected to a terminal of $i$ in $T(s)$ by a path on which $i$ does not pay for anything.

Case 3: If $T^{i}$ is not contained in $T(s)$, and $i$ does not own $s$, the edges paid for by terminals of $i$ in $T(s)$ is a single connection set.

We perform induction on the phases during which the paths $R(s)$ were generated, backwards. If $R(s)$ was generated in the last phase, this means that it is an empty path consisting only of the terminal $s$, and $T(s)=\{s\}$. The empty set here is clearly a connection set.

Let $R(s)$ and $i$ be as in Case 2, which we will prove first. Let $S$ be the edges of $T(s)$ for which $i$ pays, and consider the components of $T^{*} \backslash S$, which we want to show satisfy one of the properties of Definition 4.2. All the components that do not intersect $R(s)$ are taken care of by the inductive hypothesis, so let $C$ be a component of $T^{*} \backslash S$ that intersects $R(s)$. If $C$ contains $s$, then $C$ contains a terminal of $i$, so we are done. If $C$ contains $r(s)$, then because of the way edges of $R(s)$ are paid for, there must be some path $R(t)$ ending at a node of $R(s)$, with $t$ a terminal of $i$, and with $r(t)$ in $C$. By the inductive hypothesis, there is a path from a terminal belonging to $i$ to $r(t)$ with no edges paid for by $i$, and so there is a path to $r(s)$ with the same property, as desired. The only possibility left is that there are some edges $e_{1}$ and $e_{2}$ of $R(s)$, paid for by $i$, that border $C$ on left and right, cutting it off from the rest of $R(s)$. If $e_{1}$ and $e_{2}$ are in the same link, then by definition of a link, we have that $C$ satisfies the first property of Definition 4.2. Otherwise, by construction there must be some path $R(t)$ ending at a node of $R(s)$, with $t$ a terminal of $i$, and with $r(t)$ in $C$, and so $C$ satisfies property (2) of Definition 4.2 by the argument above.

The proofs of Cases 1 and 3 are very similar to Case 2 . The only important difference is that in Case 1 , there may 
be at most one component $C$ of $T^{*} \backslash S$ that satisfies neither property of Definition 4.2. If we take any link bordering $C$ and remove it from $S$, then $S$ becomes a connection set. This makes the set of edges paid for by $i$ be a union of at most 2 connection sets.

\section{Extensions}

Since the proof of Theorem 4.1 is constructive, it actually contains a polynomial-time algorithm for generating a 3 approximate Nash equilibrium on $T^{*}$. We can use the ideas from Theorem 3.6 to create an algorithm that given an $\alpha$ approximate Steiner forest $T$, finds a $(3+\varepsilon)$-approximate Nash equilibrium that pays for a Steiner forest $T^{\prime}$ with $c\left(T^{\prime}\right) \leq c(T)$. However, this algorithm requires a polynomialtime optimal Steiner tree finder as a subroutine. The algorithm of Theorem 4.1 generates at most 3 connection sets for each player $i$. We can check if each connection set is actually cheaper than the cheapest deviation of player $i$, which is found by the cheapest Steiner tree algorithm. If it is, then we have a $(3+\varepsilon)$-approximate Nash equilibrium. Otherwise, we can replace this connection set with the cheapest deviation tree and run this algorithm over again. If we use a 2-approximate Steiner forest $T$, and an optimal Steiner tree 1.55-approximation algorithm from [18] as our subroutine, then the above algorithm actually gives a $(4.65+\varepsilon)$ approximate Nash equilibrium on $T^{\prime}$ with $c\left(T^{\prime}\right) \leq 2 \cdot O P T$, in time polynomial in $n$ and $\varepsilon^{-1}$.

\section{Lower bounds for approximate Nash}

Claim 4.5 For any $\epsilon>0$, there is a game such that any equilibrium which purchases the optimal network is at least a $\left(\frac{3}{2}-\epsilon\right)$-approximate Nash equilibrium.

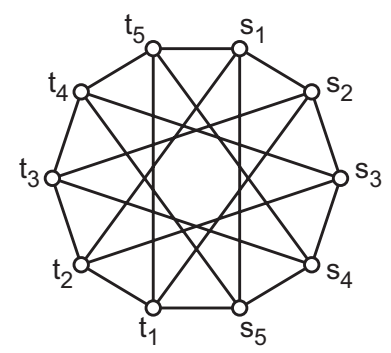

(a)

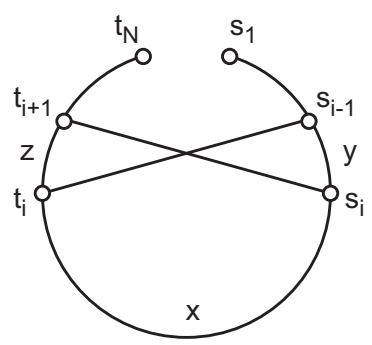

(b)
Figure 5: A game with best Nash equilibrium on OPT tending to at least a $\frac{3}{2}$-approximation.

Proof. Construct the graph $H_{N}$ on $2 N$ vertices as follows. Begin with a cycle on $2 N$ vertices, and number the vertices 1 through $2 N$ in a clockwise fashion. For vertex $i$, add an edge to vertices $i+N-1 \bmod (2 N)$ and $i+N+1 \bmod (2 N)$. Let all edges have cost 1 . Finally, we will add $N$ players with 2 terminals, $s_{i}$ and $t_{i}$, for each player $i$. At node $j$, add the label $s_{j}$ if $j \leq N$ and $t_{j-N}$ otherwise. Figure 5(a) shows such a game with $N=5$.

Consider the optimal network $T^{*}$ consisting of all edges in the outer cycle except $\left(s_{1}, t_{N}\right)$. We would like to show that any Nash that purchases this solution must be at least $\left(\frac{6 N-21}{4 N-11}\right)$-approximate. This clearly would prove our claim.
First we show that players 1 and $N$ are not willing to contribute too much to any solution that is better than $\left(\frac{3}{2}\right)$ approximate. Suppose we have such a solution. Define $x$ to be player 1's contribution to his connecting path in $T^{*}$, and define $y$ to be his contribution to the remainder of $T^{*}$. Thus player 1 has a total payment of $x+y$. Player 1 can deviate to only pay for $x$. Furthermore, player 1 can deviate to purchase only $y$ and the edge $\left(s_{1}, t_{N}\right)$. If we have a solution that is at most $\left(\frac{3}{2}\right)$-approximate, then we have that $\frac{x}{x+y} \geq \frac{2}{3}$ and similarly $\frac{y+1}{x+y} \geq \frac{2}{3}$. Taken together this implies that $\frac{1}{x+y} \geq \frac{1}{3}$, or $x+y \leq 3$. A symmetric argument shows that player $N$ is also unwilling to contribute more than 3 .

Thus we have that the remaining $N-2$ players must together contribute at least $2 N-7$. Therefore there must be some player other than 1 or $N$ who contributes $\frac{2 N-7}{N-2}$. Suppose player $i$ is such a player. Let $x$ be the amount that player $i$ contributes to his connecting path in $T^{*}$. Let $y$ be his contribution to $\left(s_{i-1}, s_{i}\right)$ and let $z$ be his contribution to $\left(t_{i}, t_{i+1}\right)$. See Figure 5(b).

Now consider three possible deviations available to player $i$. He could choose to contribute only $x$. He could contribute $y$ and purchase edge $\left(s_{i-1}, t_{i}\right)$ for an additional cost of 1 . Or he could contribute $z$ and purchase edge $\left(s_{i}, t_{i+1}\right)$, also for an additional cost of 1 . We will only consider these possible deviations, although of course there are others. Note that if $i$ was contributing to any other portion of $T^{*}$, then we could remove those contributions and increase $x, y$, and $z$, thereby strictly decreasing $i$ 's incentive to deviate. Thus we can safely assume that these are $i$ 's only payments, and hence

$$
x+y+z \geq \frac{2 N-7}{N-2} .
$$

Since $i$ is currently paying at least $x+y+z$, we know that his incentive to deviate is at least

$$
\max \left(\frac{x+y+z}{x}, \frac{x+y+z}{y+1}, \frac{x+y+z}{z+1}\right) .
$$

This function is minimized when $x=y+1=z+1$. Solving for $x$ we find that

$$
x \geq \frac{4 N-11}{3 N-6} .
$$

Thus player $i$ 's incentive to deviate is at least

$$
\frac{x+y+z}{x} \geq \frac{3 x-2}{x}=3-\frac{2}{x} \geq 3-2 \frac{3 N-6}{4 N-11}=\frac{6 N-21}{4 N-11} .
$$

Therefore as $N$ grows, this lower bound on player $i$ 's incentive to deviate tends towards $\frac{3}{2}$. Note that in this proof, we only considered one optimal network, namely $T^{*}$. If we modify $G$ by increasing the costs of all edges not in $T^{*}$ by some small $\varepsilon>0$, then $T^{*}$ is the only optimal network. Repeating the above analysis under these new costs still yields a lower bound of $\frac{3}{2}$ for the best approximate Nash on $T^{*}$ in the limit as $N$ grows and $\varepsilon$ tends to 0 .

\section{NP COMPLETENESS}

In this section, we present a brief proof that determining the existence of Nash equilibria in a given graph is NPcomplete if the number of players is $O(n)$. We present a reduction from 3-SAT to show that the problem is NP-hard. The graph constructed will have unit cost edges.

Consider an arbitrary instance of 3-SAT with clauses $C_{j}$ and variables $x_{i}$. For each variable $x_{i}$ construct the gadget 
shown in Figure 6a. When player $i$ buys the left path or right path, this corresponds to $x_{i}$ being set to be true or false, respectively. We will call $i$ a variable player.

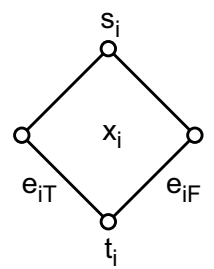

(a)

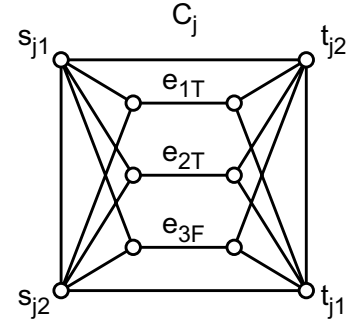

(b)
Figure 6: Gadgets for the NP-completeness reduction.

Next, for each clause $C_{j}$, construct the gadget shown in Figure 6b. Note that this example is for the case where $C_{j}=\left(x_{1} \vee x_{2} \vee \bar{x}_{3}\right)$. Furthermore, the edges labeled $e_{1 T}$, $e_{2 T}$, and $e_{3 F}$ are the same edges that appear in the variable gadgets. In other words, among all clauses and variables, there is only one edge labeled $x_{i T}$ and only one labeled $x_{i F}$. We will call both players on this gadget clause players.

Suppose that there is a satisfying assignment $A$ in our 3-SAT instance. Consider the strategy in which variable player $i$ fully buys the left path if $x_{i}$ is true in $A$ and fully buys the right path otherwise. Since this is a satisfying assignment, by our construction each clause gadget has at least one interior edge fully paid for by a variable player. For each clause $C_{j}$, let $e$ be one such edge, and let both players on this gadget buy the unique path of cost 3 that connects their terminals which uses edge $e$. It is easy to see that this forms a Nash equilibrium.

Suppose now that there is a Nash equilibrium. From our example in Figure 1, we know that if we consider any clause and look at the two corresponding players, these players must use some edges other than just those on the perimeter of the gadget. In fact, by simple case checking it is clear that in a Nash equilibrium, no edges on the perimeter of the gadget are bought at all. This implies that variable players only select paths within their gadget. Furthermore, it implies that variable players must pay fully for their entire path. Suppose $i$ is a variable player who has selected the left (true) path, but has not paid fully for the second edge in that path. The remainder of this cost must be paid for by some clause player or players. But for such a clause player to use this edge, he must also buy two other edges, which are not used by any other player. Hence such a clause player must pay strictly more than 2 . But there is always a path he could use to connect of cost exactly 2 , so this can not happen in a Nash equilibrium. Thus we have established that variable players pay fully for their own paths.

Now consider any clause gadget. Since we have a Nash equilibrium, we know that only internal edges are used. But since each clause player can connect his terminals using perimeter edges for a cost of exactly 2, one of the interior variable edges must be bought by a variable player in each clause gadget. If we consider a truth assignment $A$ in which $x_{i}$ is true if and only if player $i$ selects the left (true) path, then this obviously satisfies our 3-SAT instance, as every clause has at least one variable forcing it to evaluate to true.
Therefore, this game has a Nash equilibrium if and only if the corresponding formula is satisfiable, and since this problem is clearly in NP, determining whether a Nash equilibrium exists is NP-Complete.

\section{Two player game is solvable}

Consider a simplified two-player version of the game in which each player $i$ has only two terminals. There exists a polynomial time algorithm to find a Nash equilibrium for this game. The algorithm simply enumerates over all possible Nash equilibrium structures. Since the Nash network is always a forest with four terminals, it either has to be two disjoint paths or a tree with $T^{1} \cap T^{2}$ being a contiguous set of edges. We restrict the search space to polynomial size by using the properties of a Nash tree. For an actual description of the algorithm, see the full version of our paper [3]. Note that the complexity of finding Nash equilibria with a constant number of players greater than two remains an open problem.

\section{Acknowledgments}

This project arose in a class taught by Eric Friedman. It began as joint work with Ranjith Rajagopalan. We would like to thank him for many useful insights and contributions.

\section{REFERENCES}

[1] A. Agrawal, P. Klein, and R. Ravi. When trees collide: An approximation algorithm for the generalized Steiner problem on networks. SIAM Journal on Computing, 24(3) 445-456 (1995).

[2] A. Akella, R. Karp, C. Papadimitrou, S. Seshan, and S. Shenker. Selfish Behavior and Stability of the Internet: A Game-Theoretic Analysis of TCP. In Proceedings of SIGCOMM, 2002

[3] E. Anshelevich, A. Dasgupta, É. Tardos, and T. Wexler. Near-Optimal Network Design with Selfish Agents. See www.cs.cornell.edu/ wexler/

[4] V. Bala and S. Goyal. A Non-Cooperative Model of Network Formation. In Econometrica 68(2000), 1181-1229.

[5] M. Charikar, C. Chekuri, T. Cheung, Z. Dai, A. Goel, S. Guha. Approximation Algorithms for Directed Steiner Problems. In SODA, 1998.

[6] A. Czumaj, P. Krysta, and B. Vöcking. Selfish Traffic Allocation for Server Farms. In STOC, 287-296, 2002.

[7] A. Czumaj and B. Vöcking. Tight bounds for worst-case equilibria. In SODA, 413-420, 2002.

[8] D. Dutta A. Goel, and J. Heidemann. Oblivious AQM and Nash Equilibrium. In Proceeding of the IEEE Infocom, 2003.

[9] A. Fabrikant, A. Luthra, E. Maneva, S. Papadimitriou, and S. Shenker. On a Network Creation Game. Unpublished manuscript.

[10] U. Feige. A threshold of $\lg n$ for approximating set-cover. In STOC, 314-318, 1996.

[11] J. Feigenbaum, C. H. Papadimitriou, S. Shenker. Sharing the Cost of Multicast Transmissions. JCSS 63 (1) pages 21-41, 2001.

[12] M. Goemans, and D. Williamson. A General Approximation Technique for Constrained Forest Problems. SIAM Journal on Computing, 296-317, 1995. 
[13] H. Heller and S. Sarangi. Nash Networks with Heterogeneous Agents. Working Paper Series 2001, E-2001-1, Virginia Tech.

[14] K. Jain and V. Vazirani. Applications of Approximation Algorithms to Cooperative Games. In STOC, 2001.

[15] E. Koutsoupias and C. Papadimitriou. Worst-case equilibria. In STACS, 404-413, 1999.

[16] M. Mavronicolas and P. Spirakis. The price of selfish routing. In STOC, 510-519, 2001.

[17] C. Papadimitriou. Algorithms, Games, and the Internet. In STOC, 749-753, 2001.

[18] G. Robins and A. Zelikovsky. Improved Steiner Tree Approximation in Graphs. In SODA, 770-779, 2000.

[19] T. Roughgarden. The price of anarchy is independent of the network topology. In STOC, 2002.
[20] T. Roughgarden. Stackelberg scheduling strategies. In STOC, pages 104-113, 2001.

[21] T. Roughgarden and É. Tardos. How bad is selfish routing? In FOCS, 93-102, 2000. Full version to appear in Journal of the ACM.

[22] A. Schulz and N. Stier Moses. On the Performance of User Equilibria in Traffic Networks. To appear in SODA, 2003.

[23] S. Shenker. Making Greed Work in Networks: A Game-Theoretic Analysis of Switch Service Disciplines. In IEEE/ACM Transactions on Networking, 819-831, 1995.

[24] A. Vetta. Nash equilibria in competitive societies with applications to facility location, traffic routing and auctions. In FOCS, 2002. 